\title{
Preliminary Results on Correct-by-Construction Control Software Synthesis for Adaptive Cruise Control
}

\author{
Petter Nilsson ${ }^{1}$, Omar Hussien ${ }^{3}$, Yuxiao $\mathrm{Chen}^{2}$, Ayca Balkan $^{3}$, Matthias Rungger $^{3}$, Aaron Ames ${ }^{4}$ \\ Jessy Grizzle $^{1}$, Necmiye Ozay ${ }^{1}$, Huei Peng ${ }^{2}$, Paulo Tabuada ${ }^{3}$
}

\begin{abstract}
A plethora of driver convenience and safety automation systems are being introduced into production vehicles, such as electronic stability control, adaptive cruise control, lane keeping, and obstacle avoidance. Assuring the seamless and safe integration of each new automation function with existing control functions is a major challenge for vehicle manufacturers. This challenge is compounded by having different suppliers providing software modules for different control functionalities. In this paper, we report on our preliminary steps to address this problem through a fresh perspective combining formal methods, control theory, and correct-by-construction software synthesis. In particular, we begin the process of synthesizing the control software module for adaptive cruise control from formal specifications given in Linear Temporal Logic. In the longer run, we will endow each interacting software module with an assume-guarantee specification stating under which environment assumptions the module is guaranteed to meet its specifications. These assume-guarantee specifications will then be used to formally prove correctness of the cyber-physical system obtained where the integrated modules interact with the physical dynamics.
\end{abstract}

\section{INTRODUCTION}

Adaptive Cruise Control (ACC) is a driver assistance system designed to provide improved convenience and comfort with respect to conventional cruise control systems. When there is no preceding vehicle in sight, an ACCequipped vehicle behaves just like one with conventional cruise control, i.e., it will maintain a constant speed set by the driver. When a preceding vehicle is detected and is driving at a speed slower than the preset speed, an ACCequipped vehicle changes its control objective to maintaining a safe headway (range) or time headway instead. These two modes are commonly known as the speed (cruise) mode, and distance (following) mode, respectively, and the preceding vehicle is commonly called the lead vehicle.

ACC has been available on production vehicles since the mid-1990s. Many of the early ACC systems shut off below a given threshold speed. More recently, full-range (stopand-go) ACC is available that can bring a vehicle to a full stop and then launch from standstill, and is thus capable of dealing with congested urban traffic. In the last several years, some auto makers (e.g., Volvo and Cadillac) have introduced an automated full-braking function to leverage hardware

\footnotetext{
${ }^{1}$ Dept. of Electrical Engineering and Computer Science, ${ }^{2}$ Dept. of Mechanical Engineering, University of Michigan, Ann Arbor, MI \{pettni, chenyx, grizzle, necmiye, hpeng\}@umich.edu ${ }^{3}$ Dept. of Electrical Engineering, University of California at Los Angles, \{ohussien, abalkan, rungger, tabuada\}@ucla.edu and ${ }^{4}$ Dept. of Mechanical Engineering, Texas A\&M University, College Station, TX amesetamu. edu
}

already installed for ACC, tiptoeing the red-line between comfort/convenience systems and active safety systems. This trend is likely to continue as more automated vehicle control functions are introduced on production vehicles.

In [1], NHTSA announced its policy on the development of automated vehicles, and defined five levels of vehicle automation: Level 0 (no automation), Level 1 (functionspecific automation, such as ACC or electronic stability control), Level 2 (combined function automation, such as ACC plus lane keeping), Level 3 (limited self-driving), and finally Level 4 (full self-driving). All new production cars in the US are already at Level 1 , because electronic stability control has been required for all new cars in the US since 2012. A major challenge for control engineers is that as more automation functions are introduced (either required or as options), their seamless integration with existing control functions must be guaranteed. This requirement is important both to ensure vehicle safety and for tort/liability considerations.

This paper is the first step towards the broader objective of synthesizing correct-by-construction control software for automation Level 2 and beyond. By correct-by-construction we mean control software that is guaranteed to meet its formal specifications given certain assumptions on the physical plant and implementation platform. The formal specifications considered in this paper are given in Linear Temporal Logic, a common specification language for software systems. Here, we begin the task of synthesizing correct-by-construction control software for ACC in the special case that the lead vehicle's speed is constant. Two synthesis methods are used, one performing set computation directly on the continuous domain, and a second based on finite-state abstractions. The two resulting correct-by-construction controllers are compared by running simulations in Simulink and on a 16 degree of freedom model in CarSin 11 In subsequent work, we will address stop-and-go ACC with variable lead-vehicle speed, correct-by-construction control software for lane keeping, and correct-by-construction control software when ACC and lane keeping are simultaneously active.

\section{AdAptive CRUise Control}

For ACC systems, the most important function is to maintain a safe and comfortable range from the preceding vehicle. Whether a range is safe or not is based on metrics such as time-to-collision or deceleration needed to avoid a crash. Whether a range is comfortable or not is subjective,

${ }^{1} \mathrm{CarSim}$ is a vehicle simulation package that is widely used in industry. 
and may consider additional factors such as value and rate of change of range, time-headway, and jerk.

\section{A. Past Work}

Adaptive cruise control started as an extension of conventional cruise control (CCC), which was how it was described in relevant ISO [2] and SAE [3] standards. In these early design concepts, ACC was a phase or mode of the overall control system, and even rode-on existing CCC hardware architecture: in the ACC mode, the speed command to the CCC servo-loop was adjusted to achieve the desired range control objective (e.g., [4]). This nested control architecture resulted in slower response (in comparison to when throttle and brake are controlled directly), which was observed in prototype ACC vehicles in field tests [5]. Comparison between the velocity-command approach and acceleration command approach was analyzed in [6].

The potential of ACC-equipped vehicles for improving traffic flow and safety has been studied extensively since the 1990s [7][8]. In addition to traffic flow and safety, string stability [9][10][11][12], congestion [13], fuel economy [14] and integration with crash avoidance [15] have also been studied. An extensive survey on ACC designs, including the underlying control concepts, is given in [16]. In recent years, MPC is widely used in ACC design [17].

Although driver assistance and safety modules such as ACC have been investigated for many years, the emphasis on the correctness of a module's software implementation is much more recent. Safety verification of evasive maneuvers for autonomous vehicles is addressed in [18]. By computing the reachable set of each vehicle under different types of uncertainties and disturbances, safety is ensured whenever the reachable sets for different vehicles are disjoint. The computation of reachability sets for hybrid systems is known to be expensive and in [19] verification is done through counterexample-guided search. Rather than working with a detailed model of the system to be verified, an abstraction is used. Verifying the abstraction leads to counterexamples that may be spurious, i.e., they may not be true behaviors of the real system. However, since reachability analysis on the abstraction is cheaper, a two-step approach is taken: 1) the abstract model is used to obtain counterexamples; 2) the counterexamples are proved or disproved on a detailed model of the system to be verified. The previous approaches to verification fall in the class of model checking, given a specification one checks (by reachability computation or counterexample-guided search) if the specification is met. A different approach, considered in [20], is theorem proving. Here, one writes the assumptions about the system and its environment in a convenient logic and proves a theorem stating that the desired specification follows from the assumptions. A related approach is the use of satisfiability (SAT) and satisfiability modulo theory (SMT) solvers instead of a customized logic as was done in [21]. Unlike all the aforementioned approaches, we do not verify an existing software module. Instead, we synthesize a software module that is guaranteed to satisfy the specification by construction, hence the term correct-by-construction.

\section{B. Simplified Model For Constant Lead Vehicle Speed}

The vehicle is modeled as a (lumped) point mass $m$ moving along a straight line. The net action of braking and engine torque applied to the wheels is lumped as a net force $F_{w}$ acting on the mass of the vehicle, while the combined aerodynamic and rolling resistance is gathered into a net force $F_{r}$,

$$
m \dot{v}=F_{w}-F_{r}
$$

In the above equation, $F_{w}$ is viewed as the control input and is assumed to be bounded by

$$
-0.3 m g \leq F_{w} \leq 0.2 m g
$$

where $g$ is the gravitational constant. Such a bound is consistent with non-emergency braking and acceleration, and thus with the "driver convenience" notion of ACC. The term $F_{r}$ is represented by

$$
F_{r}=f_{0}+f_{1} v+f_{2} v^{2}
$$

We limit the admissible velocities to a bounded set $\mathcal{V}=$ $\left[v_{\min }, v_{\max }\right]$ with $v_{\min } \geq 0$.

To include a lead vehicle in the system description, we use a hybrid system model with two discrete modes $M_{1}$ and $M_{2}$, called no lead car and lead car mode, respectively. The lead car mode $M_{2}$ has an additional continuous state $h$ which measures the headway to the lead car. The continuous dynamics of $M_{1}$ are those of equation (1) while the continuous dynamics in mode $M_{2}$ contain an additional equation describing the dynamics of the headway:

$$
\begin{aligned}
m \dot{v} & =F_{w}-f_{0}-f_{1} v-f_{2} v^{2} \\
\dot{h} & =v_{L}-v
\end{aligned}
$$

The two modes have different state spaces, mode $M_{1}$ has state space $\mathcal{V}$ while mode $M_{2}$ has state space $\mathcal{V} \times \mathcal{H}$, where $\mathcal{H}=\left[0, h_{\text {max }}\right]$ for an upper limit $h_{\max }$ on the radar range.

In practice, the system is in $M_{2}$ if there is a car within the radar range, and in $M_{1}$ otherwise. Switching between the two states is governed by lane changes of lead cars, which are modeled using reset maps $R_{1,2}: \mathcal{V} \rightarrow 2^{\mathcal{V} \times \mathcal{H}}, R_{2,1}$ : $\mathcal{V} \times \mathcal{H} \rightarrow \mathcal{V}$ and $R_{2,2}: \mathcal{V} \times \mathcal{H} \rightarrow 2^{\mathcal{V} \times \mathcal{H}}$

$$
\begin{aligned}
R_{1,2}(v) & =\{(v, \bar{h}): \bar{h} \in \mathcal{H}\}, \quad R_{2,1}(v, h)=(v), \\
R_{2,2}(v, h) & =\{(v, \bar{h}): \bar{h} \in \mathcal{H}\} .
\end{aligned}
$$

Here $R_{1,2}$ models a transition from the no lead car mode $M_{1}$ to the lead car mode $M_{2}$, where the headway is initialized to some $\bar{h} \in \mathcal{H}$. Similarly, $R_{2,2}$ models situations where the radar reading suddenly changes as a result of lane changes undertaken by cars in front.

The switching is assumed to be non-deterministic, except for the case when $h$ reaches $h_{\max }$ in mode $M_{2}$, in which case a forced transition to mode $M_{1}$ occurs. The complete hybrid model is shown schematically in Fig. 1. The vehicle 


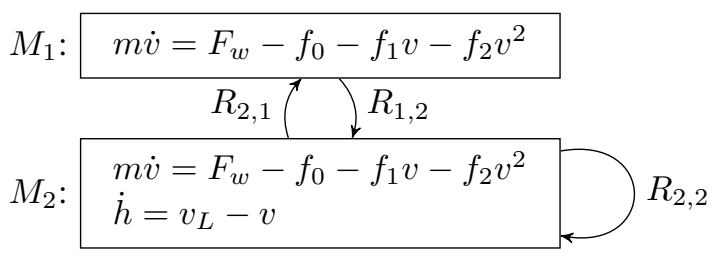

Fig. 1: Hybrid system model.

parameters ${ }^{2}$ we assume have been taken from the "S-Class Sedan" model in CarSim. We also restrict the domain to $v_{\min }=0, v_{\max }=35, h_{\min }=0, h_{\max }=300$, where the latter models the radar range.

For simplicity, we make the following assumptions:

(A.1) The velocity $v_{L}$ of the lead car is known and constant, i.e., $\dot{v}_{L}=0$. In the following we assume $v_{L}=12 \mathrm{~m} / \mathrm{s}$.

(A.2) There is at most one lead car within the radar range at all times, i.e., $R_{2,2}(v, h)=\emptyset$ for all $(v, h) \in \mathcal{V} \times \mathcal{H}$.

\section{ACC Formal Specification}

In this section we formalize the adaptive cruise control requirements using Linear Temporal Logic (LTL). Introducing the time headway, defined as $\tau=h / v$, we summarize the requirements defined by the International Organization of Standardization, see [2, Chapter 6], as follows.

1) ACC operates in two modes: the no lead car mode and the lead car mode;

2 ) in no lead car mode, a preset desired speed $v_{d e s}$ eventually needs to be reached and maintained;

3 ) in lead car mode, a desired lower bound on safe time headway $\tau_{\text {des }}$ to the lead vehicle and an upper bound on a desired velocity $v_{\text {des }}$ eventually needs to be reached and maintained; and the time headway needs to satisfy $\tau \geq 1$ at all times,

4) independently of the mode, the input constraint (2) needs to be satisfied at all times.

Note that in requirement 3 ) different choices of time or range headway are possible, see e.g. [22].

We proceed with the translation of requirements 1)-4) into LTL. The basic building blocks of an LTL specification are the so called atomic propositions. The set of atomic propositions represents the quantities necessary to express the desired behavior. For example, to be able to refer to the two modes of ACC in the specification, we introduce the atomic proposition $M_{1}$ that is satisfied when the vehicle is in no lead car mode and the atomic proposition $M_{2}$ that is satisfied when the vehicle is in lead car mode. We also introduce the atomic propositions $G_{1}, G_{2}, S_{1}$, and $S_{2}$. We use $G_{1}$ and $G_{2}$ to express requirements 2) and 3), i.e., a desired velocity $v_{\text {des }}$ and a desired lower bound on time headway $\tau_{d e s}$ as well as an upper bound on desired velocity $v_{d e s}$, should be attained (and maintained) if the vehicle satisfies $M_{1}$ and $M_{2}$, respectively. We identify $G_{1}$

\footnotetext{
${ }^{2}$ In SI units, $m=1370 \mathrm{~kg}, f_{0}=3.8 \times 10^{-3} \times m g \mathrm{~N}, f_{1}=2.6 \times$ $10^{-5} \times m g \mathrm{Ns} / \mathrm{m}, f_{2}=0.4161 \mathrm{Ns}^{2} / \mathrm{m}^{2}$.
}

$h[\mathrm{~m}]$

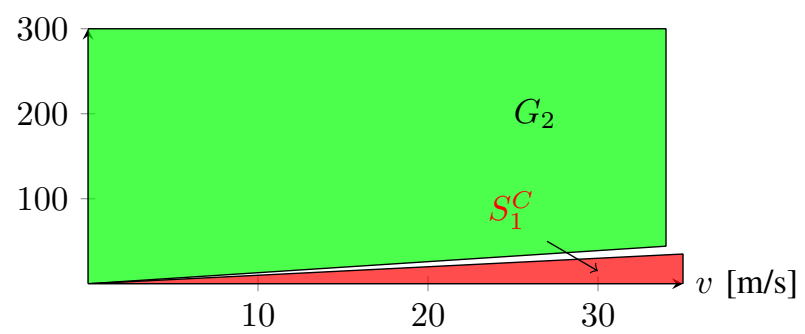

Fig. 2: State space regions for mode $M_{2}$.

and $G_{2}$ with subsets of the state space of (4) in the sense that $G_{1}$ and $G_{2}$ are satisfied whenever the state of the hybrid system belongs to the following sets:

$$
\begin{gathered}
G_{1}=\left\{v: v \in\left[v^{-}, v^{+}\right]\right\}, \\
G_{2}=\left\{(v, h): h / v \geq \tau_{\text {des }}, v \leq v^{+}, h \in \mathcal{H}\right\} .
\end{gathered}
$$

Here $v^{-}$and $v^{+}$, define the interval of tolerated deviation around the desired speed $v_{d e s}$. The set $G_{2}$ is depicted in Fig. 2. The sets $S_{1}$ and $S_{2}$ are used to express the constraints that need be satisfied at all times, i.e., the distance constraint and input constraint. These sets are given by

$$
\begin{aligned}
& S_{1}=\{(v, h): h / v \geq 1, v \in \mathcal{V}, h \in \mathcal{H}\}, \\
& S_{2}=\left\{F_{w}: F_{w} \in[-0.3,0.2]\right\} .
\end{aligned}
$$

In summary, the set of atomic propositions is given by $\left\{M_{1}, M_{2}, G_{1}, G_{2}, S_{1}, S_{2}\right\}$.

The specifications considered in this paper can be expressed using the atomic propositions, the propositional logic conjunction " $\wedge$ " and negation " $\neg$ ", and the temporal operators always " $\square$ " and eventually " $\vee$ ". Disjunction " $\vee$ " and implication " $\Rightarrow$ " can be constructed from conjunction and negation. We interpret LTL formulas over infinite sequences $(\mu, \xi, \nu)$ where the signal $\mu: \mathbb{N} \rightarrow\left\{M_{1}, M_{2}\right\}$ specifies the current mode of the system and $\xi: \mathbb{N} \rightarrow \mathbb{R}^{2}$ is a sample-and-hold trajectory of (4) given the input signal $\nu: \mathbb{N} \rightarrow \mathbb{R}$ generated by the ACC. We refer to the triple of such sequences $(\mu, \xi, \nu)$ as a behavior of the closed-loop system, i.e., (4) controlled by the ACC.

A behavior $(\mu, \xi, \nu)$ is said to satisfy $\square p$ or $\diamond p$ if the atomic proposition $p$ holds true at every time step or at some future time step(s), respectively. A closed-loop system satisfies a LTL formula $\varphi$ if every system behavior $(\mu, \xi, \nu)$ satisfies $\varphi$. For reasons of space, we do not provide the syntax and semantics of LTL and refer the interested reader to [23]. Instead, we continue with two examples. Consider the simple formulas $\varphi_{1}=\square\left(M_{1} \wedge G_{1} \wedge S_{2}\right)$ and $\varphi_{2}=\diamond\left(M_{2} \wedge G_{2}\right)$. A behavior satisfies $\varphi_{1}$ if $\mu_{t}=M_{1}$ and $\left(\xi_{t}, \nu_{t}\right) \in G_{1} \times S_{2}$ holds for all $t \in \mathbb{N}$. A behavior satisfies $\varphi_{2}$ if $\mu_{t}=M_{2}$ and $\xi_{t} \in G_{2}$ holds for some time $t \in \mathbb{N}$.

The ACC specification can be described by the LTL formula $\psi$ :

$$
\square\left(\left(M_{1} \vee S_{1}\right) \wedge S_{2}\right) \wedge \square\left(\bigwedge_{i=1}^{2} \square M_{i} \Longrightarrow \diamond \square G_{i}\right)
$$


The first term $\square\left(\left(M_{1} \vee S_{1}\right) \wedge S_{2}\right)$ ensures that the input constraint and the distance to the lead vehicle constraint are always met. Note that the distance constraint only needs to be satisfied in mode $M_{2}$, hence the disjunction $M_{1} \vee S_{1}$. We specify with the second term $\square\left(\wedge_{i=1}^{2} \square M_{i} \Longrightarrow \diamond \square G_{i}\right)$ that the desired velocity/time headway $G_{i}$ should eventually be reached and kept invariant, given that the system is in mode $M_{i}$.

Notice that the specification gives no guarantees of reaching the desired time headway and velocity, when the system switches between $M_{1}$ and $M_{2}$ infinitely often. This is in compliance with the ISO standards [2], which does not specify what the system should guarantee in such a scenario. Moreover, when the controller for mode $M_{i}$ is constructed, as long as the system is in $M_{i}$, it guarantees progress towards $G_{i}$. This property is desirable and it holds by construction because the controller cannot know if a mode change is going to occur in the future or not.

\section{Problem statement}

The goal of this paper is to synthesize a controller for the hybrid system in Figure 1 with reset maps given by (5) so that every behavior of the closed loop system satisfies the specification $\psi$ given in (10). However, if the lane-change behavior of the lead car is not properly constrained, e.g. if a car with very low speed cuts right in front of the ACCequipped car traveling at higher speed, no controller can prevent collisions, hence $\psi$ cannot be satisfied.

Motivated by this fact, the formal controller synthesis problem can now be stated as follows:

Problem 1: Assume (A.1) and (A.2) hold. Synthesize a controller and a control domain $\mathcal{D} \subseteq \mathcal{V} \times \mathcal{H}$ so that every behavior $(\mu, \xi, \nu)$ of the closed-loop system satisfies $\psi$ if the values of $R_{1,2}(v)$ are restricted to $\mathcal{D}$ for all $(v, h) \in \mathcal{V} \times \mathcal{H}$.

In what follows, we present two approaches to this problem.

Remark: Both approaches seek to synthesize controllers with control domains that are as large as possible. In general, finding a maximal $\mathcal{D}$ can be hard [24]. The presented approaches are approximate in the sense that although the synthesized controllers are provably-correct, the synthesized control domains are not necessarily maximal.

\section{Solution By COMPutations on the Continuous STATE SPACE}

In this section a solution strategy based on set computations on the continuous domain $\mathcal{V} \times \mathcal{H}$ is presented. We proceed by separately synthesizing controllers for the two modes $M_{1}$ and $M_{2}$, such that, respectively, the specifications

$$
\begin{gathered}
\diamond \square G_{1} \wedge \square S_{2} \\
\diamond \square G_{2} \wedge \square\left(S_{1} \wedge S_{2}\right)
\end{gathered}
$$

are satisfied when there is no mode switching. Then, we show how these two controllers can be implemented together to satisfy the original specification in Eq. (10) when mode switching is allowed.

\section{A. Linearized Model}

The solution strategy presented in this section relies on computation of reachable sets. Such computations are currently hard to perform for a nonlinear system and we therefore linearize the polynomial system (4) around a nominal velocity $\bar{v}$ to obtain

$$
\begin{aligned}
& \dot{v}=\frac{1}{m}\left(\bar{F}_{w}-\bar{f}_{0}-\bar{f}_{1} v\right), \\
& \dot{h}=v_{L}-v .
\end{aligned}
$$

Here $\bar{f}_{0}=f_{0}-f_{2} \bar{v}^{2}$ and $\bar{f}_{1}=f_{1}+2 f_{2} \bar{v}$. Assuming that a control input $\bar{F}_{w}$ has been computed for the linearized system (13), the modified control $F_{w}=\bar{F}_{w}+f_{2}(v-\bar{v})^{2}$ can be used to make the nonlinear system behave in accordance with the linear system. Effectively, this linearization procedure moves the nonlinearity from the system dynamics to appropriate bounds on the input. To ensure that $F_{w}$ stays inside the required range, we conservatively restrict $\bar{F}_{w}$ to the interval

$$
-0.3 m g \leq \bar{F}_{w} \leq 0.2 m g-\gamma,
$$

where $\gamma=\max _{v \in\left[v_{\min }, v_{\max }\right]} f_{2}(v-\bar{v})^{2}$. The correction term $\gamma$ is small for typical parameter values and the level of conservativeness thus not very severe. By construction, the following now holds.

Proposition 1: For any $\left(v_{0}, h_{0}\right),\left(v_{1}, h_{1}\right) \in \mathcal{V} \times \mathcal{H}$, if there exists an input $\bar{F}_{w}:[0, \tau] \rightarrow[-0.3 m g, 0.2 m g-\gamma]$ that steers the state of (13) from $\left(v_{0}, h_{0}\right)$ to $\left(v_{1}, h_{1}\right)$ in time $\tau$, then there exists an input $F_{w}:[0, \tau] \rightarrow[-0.3 m g, 0.2 m g]$ that steers the state of (4) from $\left(v_{0}, h_{0}\right)$ to $\left(v_{1}, h_{1}\right)$ in time $\tau$.

It is therefore sufficient to study reachability for the linearized system, which is described in the subsequent section.

\section{B. Backwards-Time Reachability for Linear Systems}

A polyhedral set, or polyhedron, is a set defined by linear inequalities. In this section we outline how the following problem can be solved numerically:

Problem: Given a final polyhedral set $X_{1}=\left\{x \in \mathbb{R}^{n}\right.$ : $\left.L_{x} x \leq l_{x}\right\}$, a linear time-invariant system

$$
\Xi: \quad \dot{x}=A x+B u+K,
$$

a time step $\Delta T$, and (possibly state-dependent) linear constraints on the input represented as $H_{x} x+H_{u} u \leq h_{x u}$, find an initial set $\operatorname{Pre}_{\Xi}\left(X_{1}\right) \subset \mathbb{R}^{n}$ such that for all $x_{0} \in$ $\operatorname{Pre}_{\Xi}\left(X_{1}\right)$, there exists a constant control $u_{0}$ such that

1) the control constraint $H_{x} x_{0}+H_{u} u_{0} \leq h_{x u}$ is satisfied,

2) the final set $X_{1}$ is reached at time $\Delta T$ by applying $u_{0}$, i.e. $A_{d} x_{0}+B_{d} u_{0}+K_{d} \in X_{1}$, where $A_{d}=e^{A \Delta T}$, $B_{d}=\int_{s=0}^{\Delta T} e^{A s} B \mathrm{~d} s$ and $K_{d}=\int_{s=0}^{\Delta T} e^{A s} K \mathrm{~d} s$ define the time-discretized version $\Xi_{d}$ of $\Xi$.

The constraint 1) above can be expressed as $L_{x}\left(A_{d} x_{0}+\right.$ $\left.B_{d} u_{0}+K_{d}\right) \leq l_{x}$ and combined with 2) the joint constraint on initial state and input can be written as

$$
\left[\begin{array}{cc}
L_{x} A_{d} & L_{x} B_{d} \\
H_{x} & H_{u}
\end{array}\right]\left[\begin{array}{l}
x_{0} \\
u_{0}
\end{array}\right] \leq\left[\begin{array}{c}
l_{x}-L_{x} K_{d} \\
h_{x u}
\end{array}\right] .
$$

Let $L$ be the left-hand side matrix and $l$ the right-hand side vector in (16), they define a polyhedron $P=\{(x, u) \in$ 
$\left.\mathbb{R}^{n \times m}: L\left[\begin{array}{ll}x^{T} & u^{T}\end{array}\right]^{T} \leq l\right\}$ in $x-u$-space containing all possible combinations of initial states and inputs that reach $X_{1}$. To separate out the set of all initial states, we project it onto the $x$-coordinates. If $\Pi_{x}$ is the $x$-projection operator, then $\operatorname{Pre}_{\Xi}\left(X_{1}\right)=\Pi_{x}(P)$. The projection of a polyhedron can be computed by using for example the Multi-Parametric Toolbox (MPT) [25].

\section{Finding and Steering to a Controlled-Invariant Set}

In this section we outline how to solve a problem of type

$$
\diamond \square G \wedge \square S
$$

by performing reachability calculations.

Ab equivalent condition to $\diamond \square G$ for deterministic systems is $\diamond G \wedge(G \rightarrow \square G)$, i.e. ensure that $G$ is eventually reached and that the system never leaves $G$ after reaching it for the first time. To find a strategy for staying in $G$ we search for a controlled-invariant set $C$ contained in $G$, where controlinvariance is defined as follows.

Definition 1: A set $C$ is controlled-invariant for the discrete-time system $\Xi_{d}$ if for any $x_{0} \in C$, there exists an input $u$ such that $\left(x_{0}, u\right)$ satisfies the input constraint and such that $A_{d} x_{0}+B_{d} u+K_{d} \in C$.

In the following, we propose two methods for finding a controlled-invariant set $C$ contained inside a given set $G$. Both methods rely on finding fixed points for the operator $K_{G}(C)=\operatorname{Pre}_{\Xi}(C) \cap G$.

The outside-in Algorithm 1 starts with the whole set $G$ and gradually shrinks it until only the controlled-invariant part remains. This algorithm converges to the maximal controlledinvariant set contained in $G$ [26], [27]. However, it may not terminate in a finite number of iterations and manual termination gives an over approximation of the maximal controlled-invariant set in $G$. On the other hand, if a 'small' controlled-invariant set $C^{*}$ can be found inside $G$, the insideout Algorithm 2 grows $C^{*}$ as much as possible inside of $G$. The initial 'small' controlled-invariant set $C^{*}$ can for example be some natural equilibrium point of the system. The advantage of Algorithm 2 is that the iteratively updated set $\tilde{C}$ is controlled-invariant in every iteration. It can therefore be terminated early and still produce a non-maximal controlledinvariant set inside of $G$.

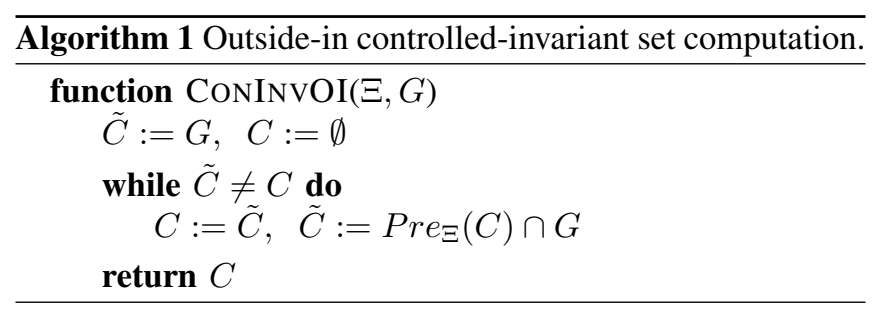

Finally, when a controlled-invariant set $C \subseteq G$ has been found, we find a list of sets $C_{2}, C_{3}, \ldots$ such that $C_{i}$ is reachable from $C_{i+1}$ using Algorithm 3 . By ensuring that all sets $C_{i}$ are contained in the safe set $S$ and denoting $C_{1}=C$, the control strategy defined as "when in $C_{i}$ go to $C_{\max (1, i-1)}$ in finite time" will satisfy (17). This control strategy can be implemented using any method capable of generating input that takes the plant to a set defined by linear inequalities. A natural choice is to use Model-Predictive Control (MPC) with a quadratic cost criterion for the discrete-time system $\Xi_{d}$, which will result in a piecewise-constant control signal for the continuous system. The domain $\mathcal{D}$ of this controller is $\cup_{i=1}^{I} C_{i}$.
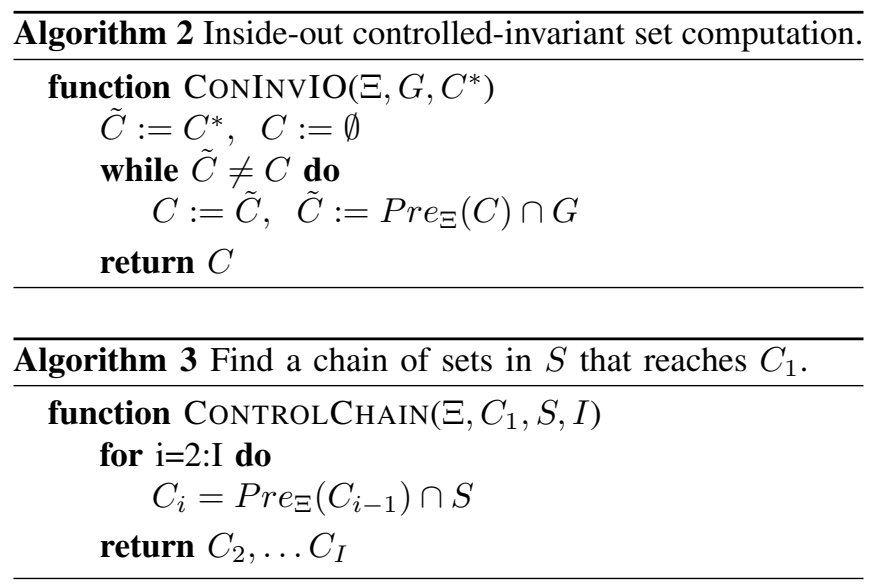

\section{Application to ACC problem}

In this section we construct controllers $K_{1}$ and $K_{2}$ for the two modes $M_{1}$ and $M_{2}$ such that the specifications (11) and 12) are satisfied. The sets of initial conditions for which these controllers satisfy their respective specifications, denoted $\mathcal{D}_{1} \subset \mathcal{V}$ and $\mathcal{D}_{2} \subset \mathcal{V} \times \mathcal{H}$, will define the necessary restrictions on the reset maps of the hybrid system.

In mode $M_{1}$ the requirement on $K_{1}$ is $\diamond \square G_{1}$, which can be interpreted as regular (non-adaptive) cruise control. It is possible to use the method presented in Sect. III-C to synthesize $K_{1}$. For simplicity, we chose to use ModelPredictive Control (MPC) to design $K_{1}$, it satisfies (11) for any trajectory starting in $\mathcal{D}_{1}=\mathcal{V}$.

Turning to controller design for mode $M_{2}$, we now apply the solution strategy in Section III-C to find a controller $K_{2}$ that satisfies 12 . If $\Xi$ denotes the linearized dynamics (13), we proceed as follows; 1$)$ call $\operatorname{ConInvOI}\left(\Xi, G_{2}\right)$ or $\operatorname{ConInviO}\left(\Xi, G_{2}, C^{*}\right)$ to find a controlled-invariant set $C_{1}$ contained in $\left.G_{2}, 2\right)$ call Control Chain $\left(\Xi, C_{1}, S_{1}, I\right)$ to find a chain of sets $C_{2}, \ldots C_{I_{1}}$ in $S_{1}$ such that $C_{i}$ is reachable from $C_{\max (1, i+1)}$.

For this example on a $3.4 \mathrm{Ghz}$ iMac, using a time step $\Delta T=0.5 s$, Algorithms 1 and 2 finish in 0.015 and 0.017 seconds, respectively, and return an over- and underapproximation of the same controlled-invariant set $C_{1} \subset G_{2}$. The call to Algorithm 3 takes 0.02 seconds on the same computer and yields two additional sets $C_{2}$ and $C_{3}$ such that $C_{\max (1, i-1)}$ is reachable from $C_{i}$. These sets are depicted in Fig. 3 .

Let $K_{2}$ be a controller that implements the control strategy 'when in $C_{i}$, go to $C_{\max (1, i-1)}$ '. By construction, $K_{2}$ then satisfies (12) for initial conditions in $\mathcal{D}_{2}=\cup_{i} C_{i}$.

Proposition 2: A controller that uses the controller $K_{1}$ when in mode $M_{1}$ and the controller $K_{2}$ when in mode $M_{2}$ 


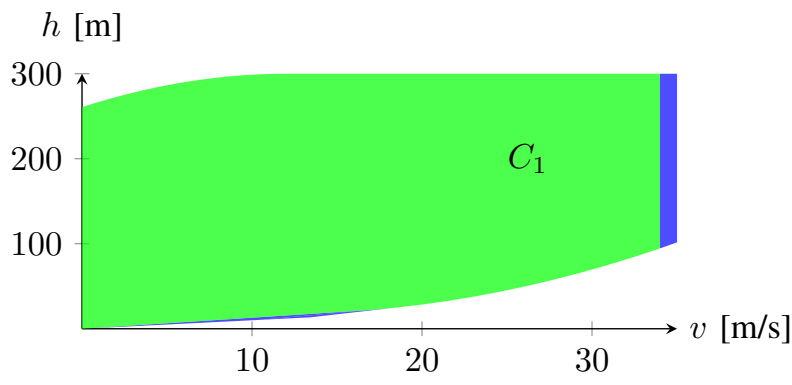

Fig. 3: The overlapping sets $C_{1} \subset C_{2} \subset C_{3}$. The control-invariant set $C_{1}$ is pictured in green and is entirely visible. $C_{2}$ and $C_{3}$, which are both subsets of $G$, are colored in blue and constitute the region from where $C_{1}$ is reachable.

solves Problem 1 with control domain $\mathcal{D}=\mathcal{D}_{2}$.

Proof: Let $\Gamma^{(v, h)}\left(\mathcal{D}_{1}\right)$ be the lifting of $\mathcal{D}_{1}$ to $\mathcal{V} \times \mathcal{H}$, that is, $\Gamma^{(v, h)}\left(\mathcal{D}_{1}\right) \doteq \mathcal{V} \times \mathcal{D}_{1}$. The result follows from the facts that $R_{2,1}\left(\mathcal{D}_{2}\right) \subseteq \mathcal{D}_{1}$ and $\mathcal{D}_{2} \subseteq \Gamma^{(v, h)}\left(\mathcal{D}_{1}\right) \cap \mathcal{D}_{2}$.

To discriminate between the control signals that correctly implement 'when in $C_{i}$, go to $C_{\max (1, i-1)}$ ', we use MPC with optimization weights that punish deviations from the desired speed or the desired time headway, depending on the current state of the system. This allows us to tune the controller in order to enhance the driving experience while still honoring the formal safety specifications.

\section{Solution via Pessoa}

In this section we describe how we compute a controller that enforces the desired behavior (10) on the system (4) using the MATLAB toolbox PESSOA [28]. The computation is based on a discrete abstraction of the system (4).

The abstraction is computed by a discretization of the state space, input space and time. The result is a finite state transition system $\Sigma=(Q, U, \delta)$, where $Q$ is the set of states, $U$ is the set of inputs and $\delta: Q \times U \rightarrow 2^{Q}$ is the transition relation. Note that $Q$ forms a grid on the state space. Therefore the sets $G_{1}, G_{2}$ and $S_{1}, S_{2}$ can easily be mapped to $Q$ by computing the grid points that fall inside the regions. We denote the corresponding sets in $Q$ as $G_{1, a}, G_{2, a}$ and $S_{1, a}, S_{2, a}$. To be able to satisfy the input constraints we set $U=S_{2, a}$.

\section{A. Controller Synthesis}

We use $\llbracket \psi \rrbracket$ to denote the states for which there exists a control strategy that enforces $\psi$. From the set $\llbracket \psi \rrbracket$ we can easily construct a strategy. For reasons of space, however, we will only discuss the computation of $\llbracket \psi \rrbracket$ and at an intuitive level.

All the controller synthesis algorithms in PESSOA are based on fixed-point solutions to certain types of games on finite graphs. Termination of these algorithms is guaranteed by the finiteness of the set of states $Q$. The interested reader is referred to [29] for further details on the algorithmic solution of such games.

To find $\llbracket \psi \rrbracket$, PESSOA first handles the safety part of the specification, i.e. $\square S_{1, a}$, by solving safety game via
Algorithm 5. The result is a set valued controller that determines the largest set of inputs that can be applied to each state while enforcing the safety specification. We then restrict the inputs of the abstraction to the inputs given by this controller and obtain a new system that will be used to compute the remaining specification via Algorithm 4. Since

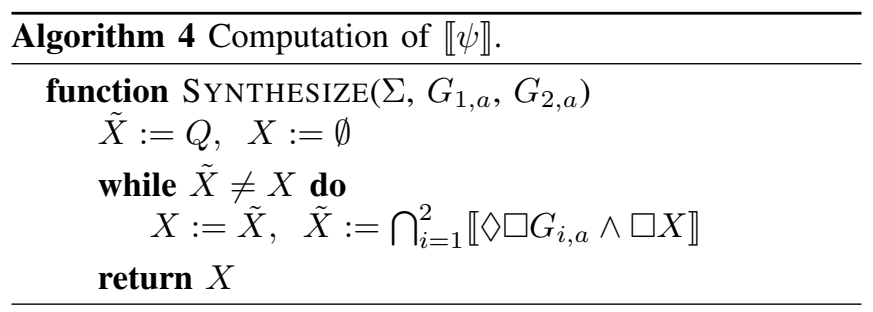

we assume that the system can change from any mode to any other mode at any time, the resulting controller should be able to enforce $\diamond \square G_{i, a}$ for $i=\{1,2\}$ at all times.

The first iteration of the algorithm computes $\bigcap_{i=1}^{2} \llbracket \diamond \square G_{i, a} \rrbracket$. These are the states from which there exist controllers that enforce $\diamond \square G_{1}$ and $\diamond \square G_{2}$. However, since the modes can change nondeterministically, this is not enough. These controllers also have to make sure that, the system always remains in the set $\bigcap_{i=1}^{2} \llbracket \triangleright \square G_{i, a} \rrbracket$. Hence, in the next iteration we add this safety constraint and compute $\bigcap_{i=1}^{2} \llbracket \diamond \square G_{i, a} \wedge \square \bigcap_{i=1}^{2} \llbracket \diamond \square G_{i, a} \rrbracket \rrbracket$. We continue the same procedure until a fixed-point is reached.

Note that at each iteration of Algorithm 4 we compute a controller which enforces $\diamond \square G_{i, a} \wedge \square K_{i-1}$, where $K_{i-1}$ is the set of states computed in iteration $i-1$. This is a "reach and stay while stay" specification which is supported in PESSOA. Again first, PESSOA handles the safety part of the specification and then it computes the domain of the controller for the "reach and stay" part via Algorithm 6. In these algorithms, we denote the predecessor operator by, $\operatorname{Pre}_{\Sigma}: 2^{Q} \rightarrow 2^{Q}$, where $\operatorname{Pre}_{\Sigma}\left(Q^{\prime}\right)=\{q \in Q \mid \exists u \in U:$ $\left.\delta(q, u) \in Q^{\prime}\right\}$.
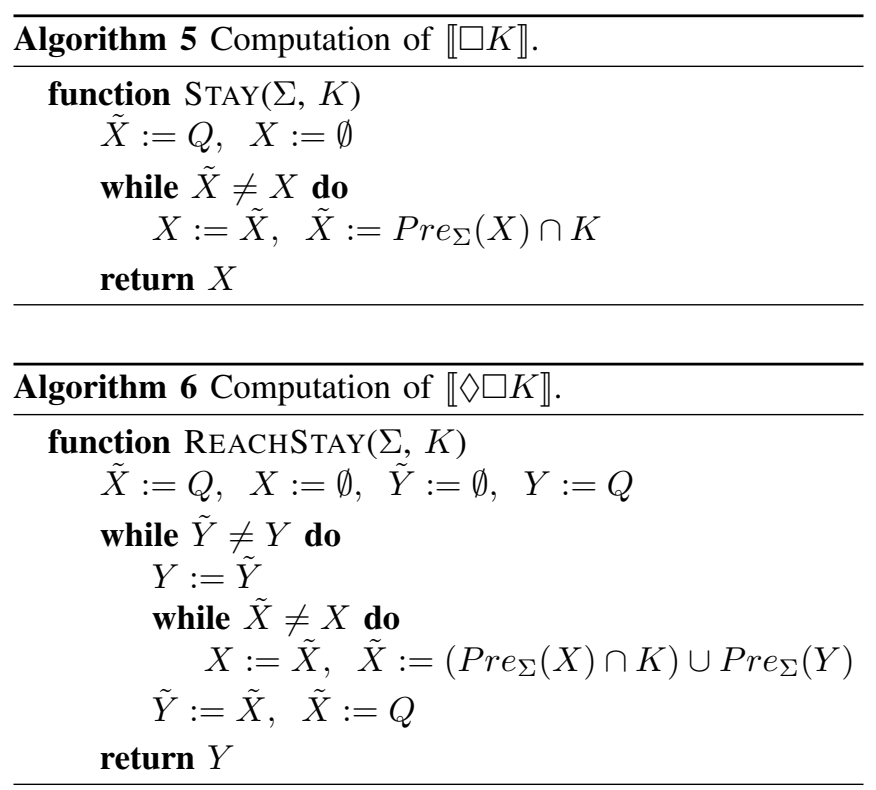


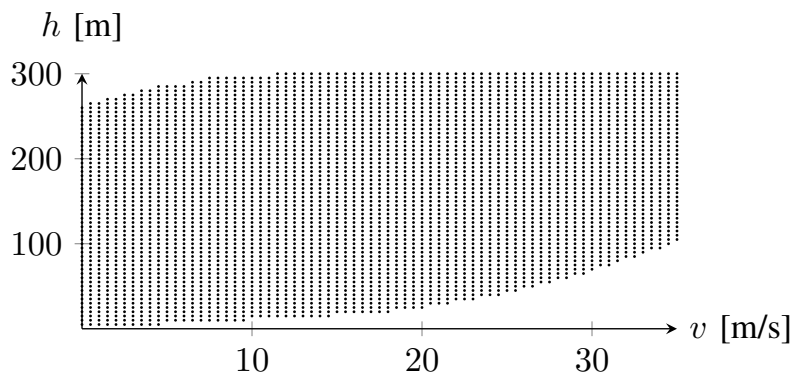

Fig. 4: The domain of the controller enforcing 10 on the system (4), i.e. $\llbracket \psi \rrbracket$. Each point represents a discrete state. $90 \%$ of the points in the $h$-coordinate have been removed for visibility.

\section{B. Solving the ACC problem}

When using PESSOA to synthesize a controller, the first step is the computation of a discrete abstraction $\Sigma$ of (4). The computation is based on the algorithms described in [30] and supported by PESSOA. For our case, we chose the state space and input space discretization parameters to be $\eta=0.5$ and $\mu=0.2$, respectively. Moreover, we use $\Delta T=0.5$ for the sampling time. Afterwards, we run the algorithms described in the previous subsection and obtain a controller that enforces the formula $\psi$ given in (10) on the system (4). It took 30.65 seconds to synthesize the controller on a 3.4 $\mathrm{GHz}$ iMac. Fig 4 illustrates the domain of the controller synthesized in PESSOA. Even though this controller was computed using the nonlinear model, its domain is comparable to the domain of the controller in Figure 3 based on a linearized model. This shows that the approximations conducted in Section 3 were not severe.

The controller synthesized by PESSOA is given by a large look-up table which is stored on the hard disk in terms of a binary decision diagram (BDD). Based on the current mode and state of the system, the BDD provides all inputs that are valid so that the closed-loop system satisfies the specification. It is straightforward to implement the controller on an embedded device, since it reduces basically to query the BDD at each sampling time. The time to query the BDD is at least two magnitudes smaller than the sampling time and therefore does not constitute a problem.

\section{Evaluation AND COMPARISON IN CARSim}

The two controllers designed in Sections III and IV have been tested in a Simulink environment using the polynomial model (4). Fig. 5a shows simulation results in the following scenario; at $t=0$ the speed of the ACC-equipped car is 5 $\mathrm{m} / \mathrm{s}$ and there is a lead car $250 \mathrm{~m}$ away which leaves the lane at $t=50 \mathrm{~s}$. A new car cuts 100 meters in front at $t=100 \mathrm{~s}$ and leaves the lane at $t=150 \mathrm{~s}$. As can be seen, the formal requirements on input, velocity and time headway are satisfied for the two modes.

The controller from section III has also been tested in CarSim, as are shown in Fig. 5b A video is also available at http://web.eecs.umich.edu/cpswiki/ public_media/

\section{DiscusSION}

We made some simplifying assumptions with regard to the lead car speed (A.1) and the number of cars within the radar range (A.2). Next, we discuss implications of these assumptions and how they can be relaxed.

A cruise controller that has practical value must be able to handle a lead car that changes speed. Relaxing the assumption (A.1) introduces environmental uncertainty into the system and increases the dimension of the state space from two to three. The theory of approximate finite-state abstractions, upon which PESSOA is based, already supports adversarial environments such as a lead car with changing velocity. Similarly, for the invariant-set based approach, the notions of a controlled-invariant set and reachability can be replaced with those of a robustly controlled-invariant set and "robust" reachability for systems with uncertainty. The increase in the dimension of the model, from two to three states, will render the synthesis of a controller in PESSOA or based on invariant-sets more challenging from a computational point of view.

On the other hand, the assumption (A.2), is rather technical and it is adopted to avoid the pathological cases where lead cars cut into the lane infinitely often in a way to prevent maintaining the desired time headway while always in mode $M_{2}$. The synthesized controllers are still correct when this assumption is relaxed to either (i) $R_{2,2}(v, h)=\mathcal{D}$ for all $(v, h) \in \mathcal{V} \times \mathcal{H}$ and there are finitely many resets, or (ii) $R_{2,2}(v, h)=G_{2}$ for all $(v, h) \in \mathcal{V} \times \mathcal{H}$.

\section{CONCLUSIONS}

In this paper, we formalized the ACC problem using a hybrid dynamical system model with two modes and an LTL specification. Then, we presented two solution approaches to synthesize correct-by-construction control software for ACC. Both approaches rely on a fixed-point-based characterization of the LTL specification, one computing such fixed-points directly on the continuous state-space, the other on a finitestate abstraction of the nonlinear dynamics.

It can happen that an ACC system cannot maintain safety within specified limits on braking (e.g., (2)), such as in the event of a car cutting dangerously close into a lane. In this case, the ACC system is expected to alert the driver through automated emergency braking and/or other feedback cues, and hand control of the vehicle back to the driver. An advantage of the presented formal methods is that the safe set (i.e., control domain $\mathcal{D}$ ) is explicitly computed and thus conditions for passing control to an emergency braking module are clearly defined.

\section{ACKNOWLEDGMENT}

The work is supported by NSF Contract \#CNS-1239037. CarSim is a registered trademark of Mechanical Simulation Corporation, Ann Arbor, MI. 

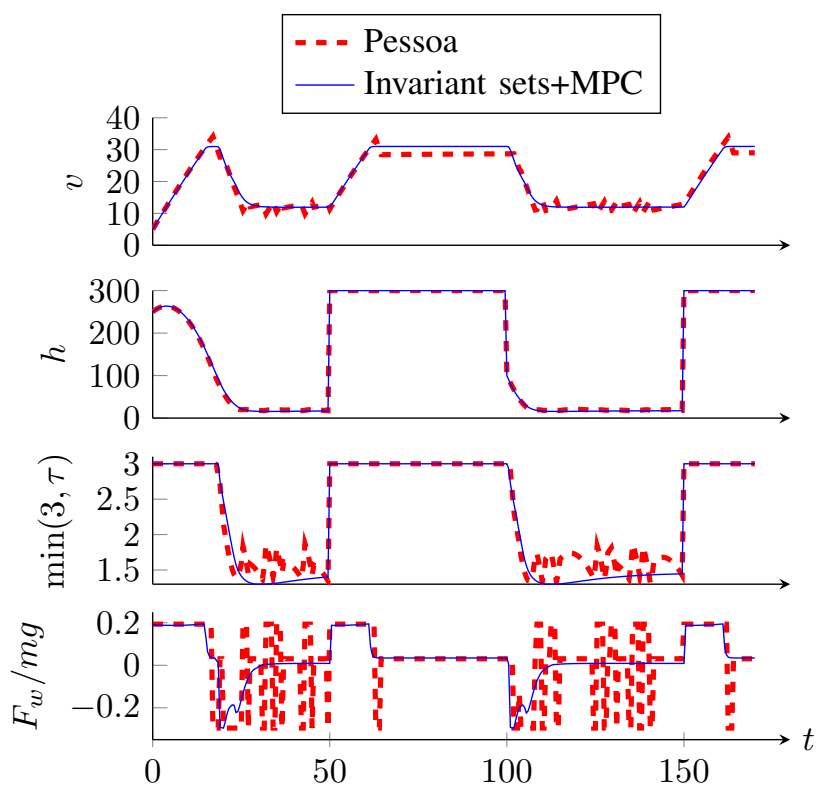

(a)
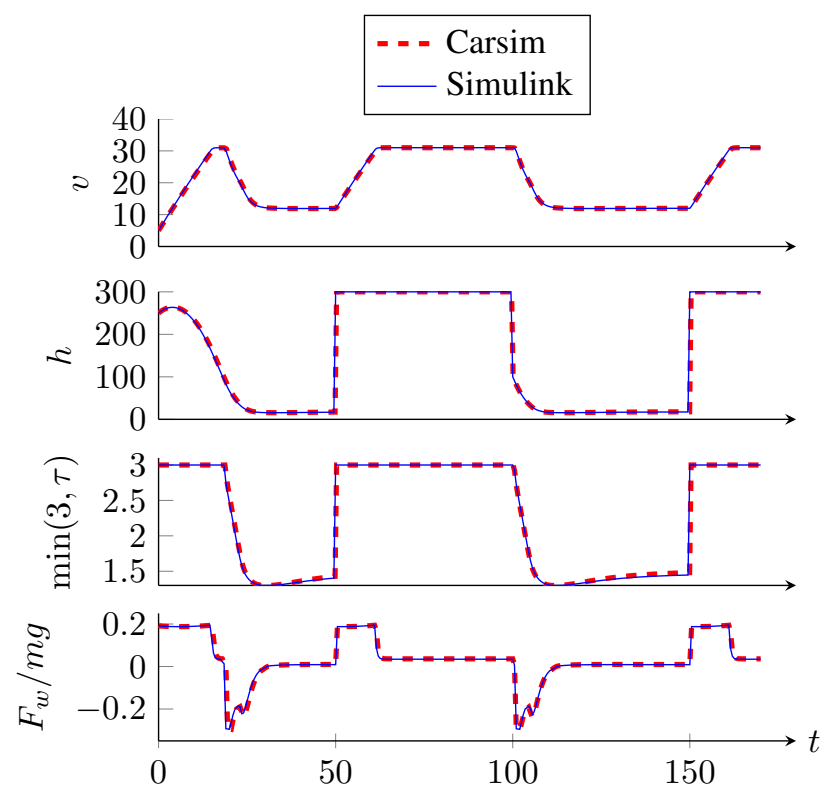

(b)

Fig. 5: Simulation results for (a) both controllers in Simulink and (b) the controller from Section III in Simulink and CarSim. The plots show, from top to bottom: speed, headway, time headway, and normalized applied wheel force.

\section{REFERENCES}

[1] (2014) National Highway Traffic Safety Administration (NHTSA). [Online]. Available: http://www.nhtsa.gov/staticfiles/rulemaking/pdf/ Automated_Vehicles_Policy.pdf

[2] ISO 15622:2010 (E), "Intelligent transport systems - adaptive cruise control systems - performance requirements and test procedures," International Organization for Standardization, Tech. Rep., 2010.

[3] SAE J2399, "Adaptive cruise control (ACC) human factors: Operating characteristics and user interface," SAE, Tech. Rep., 2003.

[4] P. Fancher, H. Peng, Z. Bareket, C. Assaf, and R. Ervin, "Evaluating the influences of adaptive cruise control systems on the longitudinal dynamics of strings of highway vehicles," Vehicle Syst. Dyn., vol. 37, pp. 125-136, 2003.

[5] DOT HS 808 849, "Intelligent cruise control field operational test," US DOT, Tech. Rep., 1998.

[6] J. Zhou and H. Peng, "String stability conditions of adaptive cruise control algorithms," in IFAC Symp. on Advances in Automotive Control, 2004.

[7] P. A. Ioannou and C.-C. Chien, "Autonomous intelligent cruise control," IEEE Trans. Veh. Tehnol., vol. 42, no. 4, pp. 657-672, 1993.

[8] B. Van Arem, C. J. van Driel, and R. Visser, "The impact of cooperative adaptive cruise control on traffic-flow characteristics," IEEE Trans. Intell. Transp. Syst., vol. 7, no. 4, pp. 429-436, 2006.

[9] C.-Y. Liang and H. Peng, "Optimal adaptive cruise control with guaranteed string stability," Vehicle Syst. Dyn., vol. 32, no. 4-5, pp. 313-330, 1999.

[10] J. Zhou and H. Peng, "Range policy of adaptive cruise control vehicles for improved flow stability and string stability," IEEE Trans. Intell. Transp. Syst., vol. 6, no. 2, pp. 229-237, 2005.

[11] X. Lu and J. Hedrick, "Practical string stability," in IAVSD Symp. on Dynamics of Vehicles on Roads and Tracks, 2003, pp. 25-29.

[12] Y. Yamamura, Y. Seto, H. Nishira, and T. Kawabe, "An ACC design method for achieving both string stability and ride comfort," J. System Design and Dynamics, vol. 2, pp. 979-990, 2008.

[13] A. Kesting, M. Treiber, M. Schönhof, and D. Helbing, "Adaptive cruise control design for active congestion avoidance," Transpn Res.C: Emerging Technologies, vol. 16, no. 6, pp. 668-683, 2008.

[14] S. Li, K. Li, R. Rajamani, and J. Wang, "Model predictive multiobjective vehicular adaptive cruise control," IEEE Trans. Control Syst. Technol., vol. 19, no. 3, pp. 556-566, 2011.

[15] S. Moon, I. Moon, and K. Yi, "Design, tuning, and evaluation of a full-range adaptive cruise control system with collision avoidance,' Control Eng. Pract., vol. 17, no. 4, pp. 442-455, 2009.

[16] A. Vahidi and A. Eskandarian, "Research advances in intelligent collision avoidance and adaptive cruise control," IEEE Trans. Intell. Transp. Syst., vol. 4, no. 3, pp. 143-153, 2003.

[17] G. Naus, J. Ploeg, M. Van de Molengraft, W. Heemels, and M. Steinbuch, "Design and implementation of parameterized adaptive cruise control: An explicit model predictive control approach," Control Eng. Pract., vol. 18, no. 8, pp. 882-892, 2010.

[18] M. Althoff, D. Althoff, D. Wollherr, and M. Buss, "Safety verification of autonomous vehicles for coordinated evasive maneuvers," in IEEE Intelligent Vehicles Symposium, 2010, pp. 1078-1083.

[19] O. Stursberg, A. Fehnker, Z. Han, and B. H. Krogh, "Verification of a cruise control system using counterexample-guided search," Control Eng. Pract., vol. 12, no. 10, pp. 1269-1278, 2004.

[20] S. M. Loos, A. Platzer, and L. Nistor, "Adaptive cruise control: Hybrid, distributed, and now formally verified," in FM 2011: Formal Methods. Springer, 2011, pp. 42-56.

[21] M. Asplund, A. Manzoor, M. Bouroche, S. Clarke, and V. Cahill, "A formal approach to autonomous vehicle coordination," in $F M$ 2012: Formal Methods. Springer, 2012, pp. 52-67.

[22] K. Vogel, "A comparison of headway and time to collision as safety indicators," Accident Anal. Prev., vol. 35, no. 3, pp. 427-433, 2003.

[23] M. Y. Vardi, "An automata-theoretic approach to linear temporal logic," in Logics for Concurrency. Springer, 1996, pp. 238-266.

[24] T. A. Henzinger, P. W. Kopke, A. Puri, and P. Varaiya, "What's decidable about hybrid automata?" in Proc. of the ACM Symp. on Theory of Computing, 1995, pp. 373-382.

[25] M. Herceg, M. Kvasnica, C. N. Jones, and M. Morari, "MultiParametric Toolbox 3.0," in Proc. of the ECC, 2013.

[26] D. Bertsekas, "Infinite time reachability of state-space regions by using feedback control," IEEE Trans. Autom. Control, vol. 17, no. 5, pp. 604-613, 1972.

[27] F. Blanchini, "Set invariance in control," Automatica, vol. 35, no. 11, pp. 1747-1767, 1999.

[28] M. Mazo Jr, A. Davitian, and P. Tabuada, "Pessoa: A tool for embedded controller synthesis," in Computer Aided Verification. Springer, 2010, pp. 566-569.

[29] W. Thomas, "On the synthesis of strategies in infinite games," in Lecture Notes in Computer Science. Springer, 1995, pp. 1-13.

[30] M. Zamani, G. Pola, M. Mazo, and P. Tabuada, "Symbolic models for nonlinear control systems without stability assumptions," IEEE Trans. Autom. Control, vol. 57, no. 7, pp. 1804-1809, 2012. 